\title{
Aluminum Corrosion Inhibition by 7-(Ethylthiobenzimidazolyl) Theophylline in 1M Hydrochloric Acid: Experimental and DFT Studies
}

\author{
N'guessan Yao Silvère Diki $^{1}$, Gbe Gondo Didier Diomandé ${ }^{2}$, Sagne Jacques Akpa ${ }^{2}$, Augustin Ouédraogo ${ }^{1}$, \\ Lemeyonouin Aliou Guillaume Pohan ${ }^{3}$, Paulin Marius Niamien ${ }^{1}$ and Albert Trokourey ${ }^{1}$. \\ ${ }^{1}$ Laboratoire de Chimie Physique, Université Félix Houphouët-Boigny, 22BP 582 Abidjan 22, Côte d'Ivoire \\ ${ }^{2}$ Laboratoire de Chimie Organique et Substances Naturelles, Université Félix Houphouët-Boigny, 22BP 582 Abidjan 22, Côte \\ d'Ivoire \\ ${ }^{3}$ UFR Sciences Biologiques, Peleforo Gon Coulibaly University, BP 1328 Korhogo, Côte d'Ivoire
}

\begin{abstract}
Inhibitive properties of 7-(ethylthiobenzimidazolyl) theophylline (7-ETBT) molecule for the corrosion of aluminum (Al) in one molar hydrochloric acid medium have been investigated using mass loss and Tafel polarization methods. The results suggest spontaneous and predominant physical adsorption of 7-ETBT on the metal surface which obeys Langmuir isotherm model. The studied inhibitor showed excellent inhibition efficiency up to $94.07 \%$ at $298 \mathrm{~K}$ for its optimum concentration $(2 \mathrm{mM})$. Moreover, Tafel polarization method revealed that 7-ETBT is a cathodic-type inhibitor. Further investigation on the morphology using scanning electron microscopy (SEM) has confirmed the existence of a protective film of inhibitor molecules on aluminum surface. Theoretical approach for the performance of 7-ETBT as Al corrosion inhibitor is also done using Density Functional Theory (DFT) level with B3LYP exchange correlation functional for 6-31G $(\mathrm{d}, \mathrm{p})$ basis set. The results provided by this theoretical study are consistent with the experimental data. All the results have shown an evidence of the effectiveness of the inhibitive effect of 7-ETBT on Al.
\end{abstract}

Key words: 7-(ethylthiobenzimidazolyl) theophylline, DFT, Corrosion, mass loss; SEM, Tafel polarization

\section{INTRODUCTION}

Owing to their high technological value and wide range of industrial applications especially in aerospace, automotive, building and household industries of aluminum and its alloys, several researchers have devoted great efforts to study their corrosion inhibition. Although aluminum and its alloys seem safe by the formation of a compact, adherent passive oxide film for its corrosion immunity in various environments, they are prone to corrode when exposed to high concentrations of acids or bases (Fouda et al., 2000; Hurlen et al., 1984). In order to reduce the corrosion of metals, several techniques have been applied. Among these techniques, the use of corrosion inhibitors is the most effective and practical way (Umoren et al., 2009).

However many corrosion inhibitors used in aqueous heating and cooling systems are generally toxic (F. et al., 2001). This toxic property limits the field of their applica- tions due to the increased awareness towards environmental pollution. The new trend is the development of organic corrosion inhibitors which are available, low-cost and mostly non-toxic (Ajmal et al., 1994; Desai et al., 1986; El-Maksoud, 2002). To be effective, an inhibitor must interact with anodic or cathodic reaction sites to delay the oxidation and reduction corrosion reaction, and prevent corrosion-active species on the surface (Obot et al., 2011). Most of the efficient inhibitors are organic compounds that contain mainly, nitrogen, sulfur, oxygen or phosphorus atoms and aromatic ring in their structure (Badr, 2009; Laarej et al., 2010) which are helpful to form complexes with metal ions. These complexes constitute a film barrier which separates the metal from the aggressive environment.

In order to assess the effectiveness of the inhibition effect of the molecule, quantum chemistry calculations are used. The most used of quantum chemical methods for corrosion 
inhibitor evaluation (Cruz et al., 2005; Khaled, 2010; Oguzie et al., 2011a) is density functional theory (DFT). This method (Cruz et al., 2004; Oguzie et al., 2011b) leads to the ground state properties of a many-electron system by using functional (Ehouman et al., 2016). Nowadays, quantum chemical calculations are widely used to study or predict reaction mechanisms and to interpret the experimental results (Lukovits et al., 1997). To get insight into chemical reactivity and selectivity of organic molecules, researchers use DFT parameters including global parameters such as electronegativity $\chi$ (Parr et al., 1978), hardness $\eta$ (Parr and Pearson, 1983), softness S (Berkowitz et al., 1985), electrophilicity index $\omega$ (Parr and Pearson, 1983). The aim of the present paper is to evaluate the behavior of 7-(ethylthiobenzimidazolyl) theophylline (Scheme 1) as an inhibitor of aluminum corrosion in $1 \mathrm{M} \mathrm{HCl}$, by analyzing both experimental data and theoretical parameters.<smiles>Cn1c(=O)c2c(ncn2CCSc2nc3ccccc3[nH]2)n(C)c1=O</smiles>

Scheme 1: Chemical structure of 7-(ethylthiobenzimidazolyl) theophylline

\section{MATERIAL AND METHODS}

\subsection{Aluminum specimen}

The samples of aluminum used in this study were in the form of rods with $10 \mathrm{~mm}$ as length and $2.5 \mathrm{~mm}$ as diameter which is the type AA 1060 and purity of $99.6 \%$.

\subsection{Reagents}

The structure of 7-ETBT has $\mathrm{C}_{16} \mathrm{H}_{16} \mathrm{~N}_{6} \mathrm{O}_{2} \mathrm{~S}$ as formula. The molecule of 7-ETBT of beige color was provided by the Laboratory of Organic Chemistry and Natural Substances, Felix Houphouet-Boigny University. Its molecular structure was identified by $1 \mathrm{H}$ NMR and $13 \mathrm{C}$ NMR spectroscopy:

- $\quad \mathrm{RMN1H}(400 \mathrm{MHz}, \mathrm{DMSO}-\mathrm{d} 6, \delta$ ppm):3.22 (s, 6H, -CH3-); 3.74 (dd, J=6.6, 5.1 Hz, 2H, N-CH2-); 4.60 (dd, 2H, -CH2-S); 7.04 (dd, 2H, HAr); 7.32 (dd, 2H, HAr); 7.96 (s, 1H, N=CH-).

- $\quad \mathrm{RMN} 13 \mathrm{C}$ (DMSO, ठ ppm): =27.56 (CH3-); 29.22 (CH3-); 32.00 (N-CH2-); 46.49 (-CH2-S); 150 (CAr); 149.34 (CAr); 148.34 (CAr).

The corrosive aqueous solution of $1 \mathrm{M} \mathrm{HCl}$ was prepared by dilution of analytical grade $37 \% \mathrm{HCl}$ purchased from Sigma-Aldrich Chemicals with bi-distilled water. Acetone of purity $99.5 \%$ was also purchased from SigmaAldrich Chemicals.

\subsection{Solution preparation}

$1 \mathrm{M} \mathrm{HCl}$ solutions without or with different concentrations of 7-ETBT ranging from 0.02 to $2 \mathrm{mM}$ were then prepared.

\subsection{Mass loss measurements}

Each test was conducted in $50 \mathrm{~mL}$ aerated and unstirred $1 \mathrm{M} \mathrm{HCl}$ solution without or with the desired concentrations of the tested inhibitor for $1 \mathrm{~h}$ immersion time at different temperatures by using a water controlled thermostat (Memmert, precision $\pm 0.5{ }^{\circ} \mathrm{C}$ ). Prior, the aluminum samples were polished with different emery papers, washed thoroughly with bi-distilled water, degreased and dried with acetone. The obtained samples were then kept in an oven (Memmert) at $70{ }^{\circ} \mathrm{C}$ and weighed accurately. After $1 \mathrm{~h}$ of immersion in the corrosive media with or without 7-ETBT, the specimens were carefully washed in bi-distilled water, dried and reweighed accurately. Triplicate experiments were performed in each case and the mean value of the mass loss was reported. From the mass loss measurements, the corrosion rate $(W)$, degree of surface coverage $(\theta)$, and inhibition efficiency (IE) were calculated using Equation 1-3 respectively (Khaled, 2008):

$$
\begin{aligned}
& W=\frac{\Delta m}{S t} \\
& \theta=\frac{W_{0}-W}{W_{0}} \\
& I E(\%)=\left(\frac{W_{0}-W}{W_{0}}\right) * 100
\end{aligned}
$$

Where,

$\mathrm{W}_{0}$ and $\mathrm{W}$ (expressed in $\mathrm{mg} \mathrm{h}^{-1} \mathrm{~cm}^{-2}$ ) are respectively the corrosion rate without and with 7-ETBT, $\Delta \mathrm{m}$ is the mass loss, $\mathrm{S}$ is the total surface of the aluminum specimen and $\mathrm{t}$ is the immersion time.

\subsection{Electrochemical measurement}

The measurements were performed with an Autolab PGSTAT 20 potentiostat (Ecochemie, Utrecht Netherlands) controlled by GPES 4.4 software. Origin version 6.0 Software was used for plotting, graphing and fitting data. The electrochemical measurements were performed in a typical three-compartment glass cell consisting of the aluminum rod (treated as describe earlier) as working electrode (WE) with the exposed surface of $0.884 \pm 0.001$ $\mathrm{cm}^{2}$, a rolled platinum rod as counter electrode (CE), and a saturated calomel electrode (SCE) as the reference electrode. Prior to the electrochemical measurement, the working electrode was immersed in test solution 


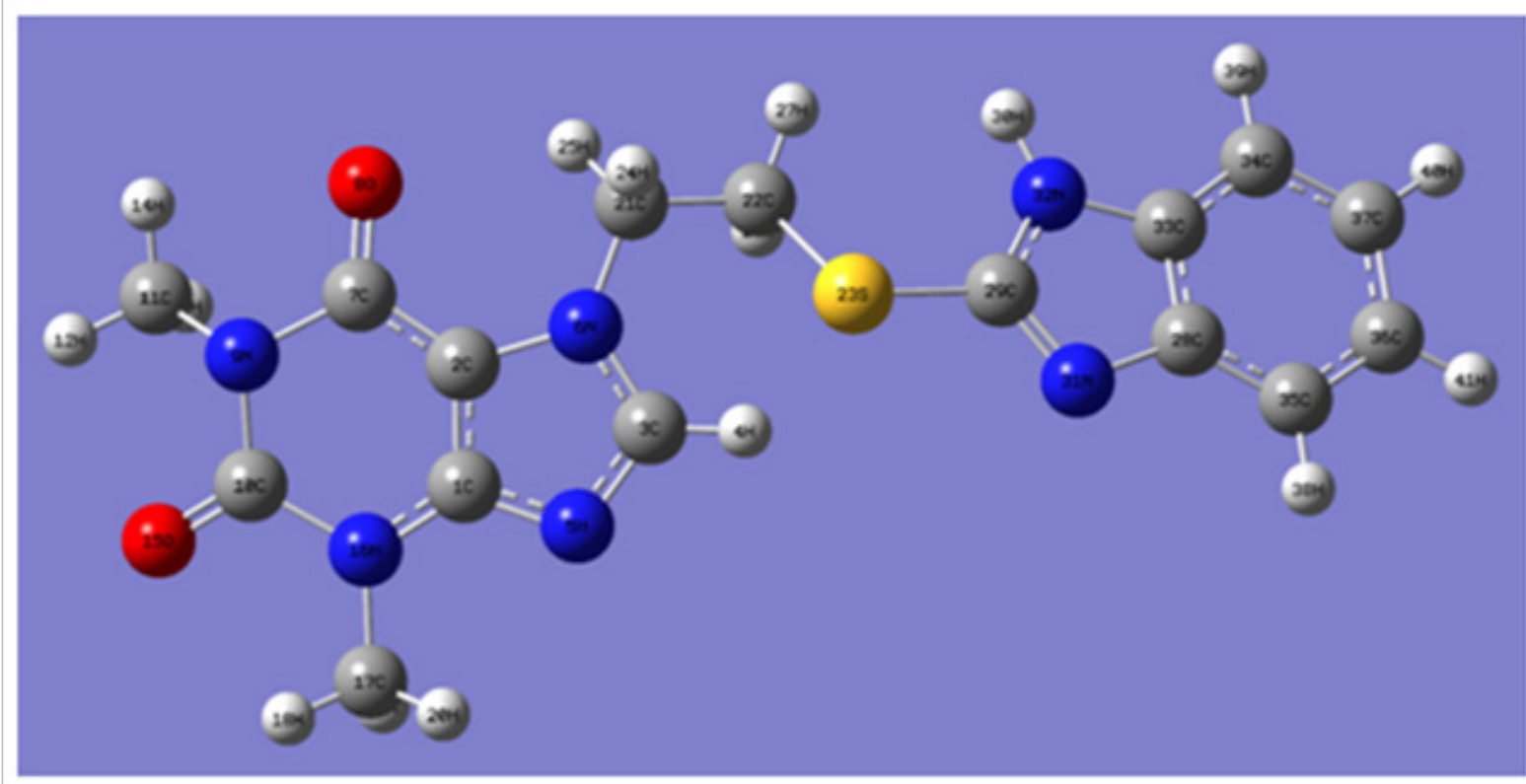

Figure 1: Optimized structure of 7-ETBT by B3LYP/6-31G (d, p).

for approximately $30 \mathrm{~min}$ until a steady open-circuit potential (OCP) was reached (Singh et al., 2016). All the experiments were conducted at room temperature (298 K). The electrolyte solution preparations were similar to those used for mass loss measurements. Tafel curves were obtained by changing the electrode potential automatically from $-1500 \mathrm{mV} / \mathrm{SCE}$ to $+500 \mathrm{mV} / \mathrm{SCE}$ versus open-circuit potential with a scan rate of $1 \mathrm{mV} / \mathrm{s}$. In each measurement, a fresh working electrode was used and the experiments were carried out in triplicate to obtain reproducible data. The inhibition efficiency (IE \%) was calculated using Equation 4:

$$
I E(\%)=\left(\frac{j_{c o r r}^{0}-j_{c o r r}^{i n h}}{j_{c o r r}^{0}}\right) \times 100
$$

Where,

$j_{\text {corr }}^{0}$ and $j_{\text {corr }}^{\text {inh }}$ are referred to the corrosion current density without and with the addition of the inhibitor, respectively.

\subsection{Surface characterization}

The scanning electron microscopy (FEG-SEM, SUPRA 40VP, ZEISS, Germany) was used to study the morphology of the aluminum surface after its treatment in the presence or absence of 7-ETBT for $1 \mathrm{~h}$ immersion at room temperature. Comparison was made between the bare sample and the immersed ones.

\subsection{Computational details}

Theoretical approach was needed to correlate experimental data and the electronic properties of the studied inhibitor. In this way, density functional theory (DFT) cal- culations were performed using the Lee-Yang-Parr (LYP) functional (Lee et al., 1988) implemented in Gaussian 09 $\mathrm{W}$ program package (Frisch et al.). This method is referred as B3LYP/6-31 G $(d, p)$ in the standard nomenclature. The geometry of the studied molecule was optimized without symmetry constraints. The aim of the present work is to highlight the relationship between the computed quantum chemical parameters and the experimentally determined inhibition efficiency of 7-ETBT against aluminum corrosion in $1 \mathrm{M} \mathrm{HCl}$. Figure 1 gives the optimized chemical structure of 7-ETBT.

\section{RESULTS AND DISCUSSION}

\subsection{Mass loss measurements}

\subsubsection{Effect of concentration and temperature on corrosion rate}

Mass loss studies were performed in the absence and presence of 7-ETBT. The concentration in that molecule ranges from 0.02 to $2 \mathrm{mM}$. The temperature varies from 298 to 318 K. As displayed in Figure 2A\&B, the increase in 7-ETBT concentration is accompanied by a decrease in corrosion rate (Figure 2A) and increase in inhibition efficiency up to $94.07 \%$ for $2 \mathrm{mM}$ of 7 -ETBT at $298 \mathrm{~K}$ (Figure 2B). These results suggest that 7-ETBT acts as an effective inhibitor of aluminum corrosion in $1 \mathrm{M} \mathrm{HCl}$ over the concentration range studied.

For a given temperature, inhibition efficiency increases with the inhibitor concentration. This behavior could be explained by the formation of a protective film which separates aluminum from the corrosive medium.

The inhibition efficiency of the studied compound during the temperature variation was also studied over 

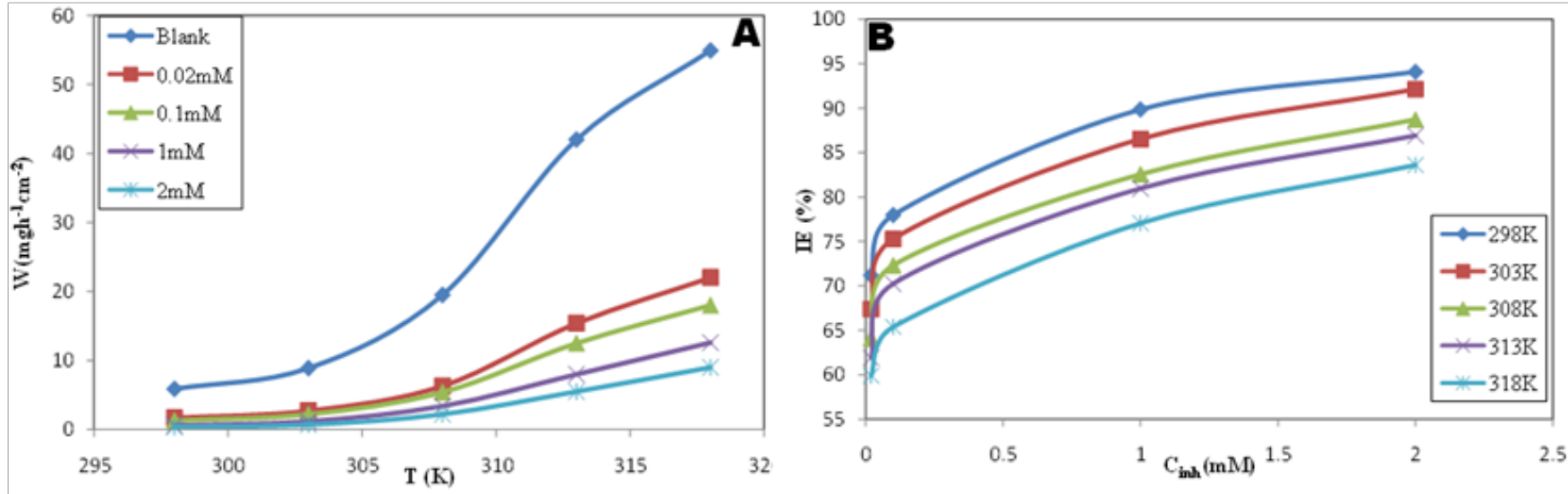

Figure 2: A) Evolution of corrosion rate with temperature for different concentrations of 7-ETBT and B) Inhibition efficiency versus inhibitor concentration at different temperatures.

the inhibitor concentration range. The evolution of inhibition efficiency versus temperature is plotted in Figure 3A. As shown in Figure 3A, the inhibition efficiency decreases with the rise in temperature for the concentration range studied. According to the literature (Saratha and Meenakshi, 2010) the decrease in inhibition efficiency with increase in temperature indicates that the process of adsorption of the inhibitor on the corroding metal surface is physisorption. This effect can be also attributed to the increase in the solubility of the protective films and of any reaction products precipitated on the surface of the metal (Santhini and Jeyaraj, 2012) thereby increasing the metal surface exposure to corrosive attack when the temperature rises.

\subsubsection{Adsorption isotherms and thermodynamic parameters}

A determination of the type of adsorption isotherm itself provides useful information on the adsorption process such as surface coverage, adsorption equilibrium constant and information on the interaction between the organic compound and the metal surface (Desimone et al., 2011). In order to obtain the suitable adsorption isotherm, attempts were made to fit the $\Theta$ values to various

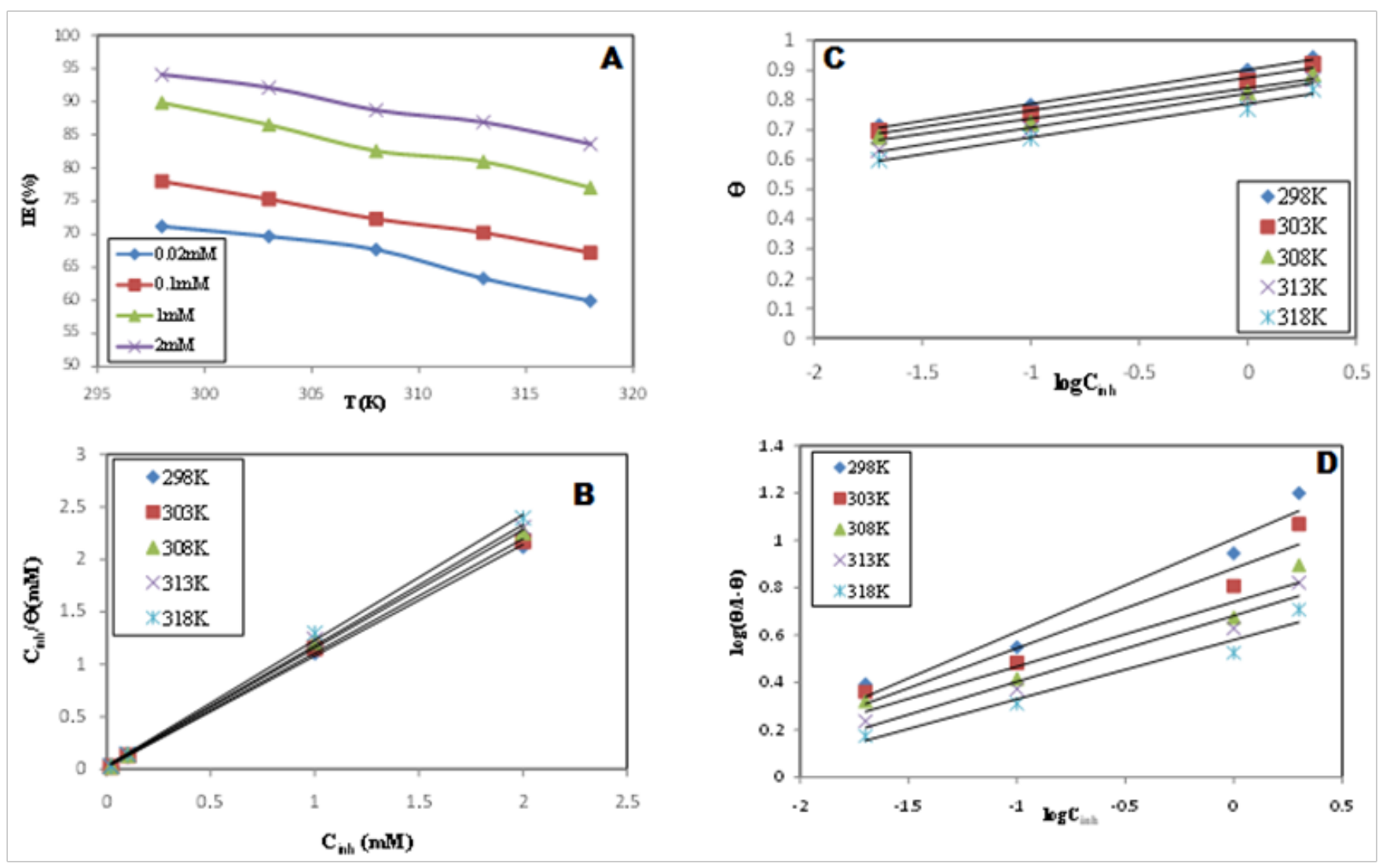

Figure 3: A) Inhibition efficiency versus temperature for different concentrations, B) Langmuir, C) Temkin and

D) El-Awady adsorption isotherms for different temperatures. 
adsorption isotherms namely Langmuir, Temkin, and El-Awady isotherm (Equation 5-7 respectively):

$$
\begin{aligned}
& \frac{C_{i n h}}{\theta}=\frac{1}{K_{a d s}}+C_{i n h} \\
& \theta=\frac{2.303}{f}\left[\log K_{a d s}+\log C_{i n h}\right] \\
& \log \left(\frac{\theta}{1-\theta}\right)=\log K+y \log C_{i n h}
\end{aligned}
$$

Where, $C_{i n h}$ is 7-ETBT concentration, $K_{a d s}$ is the equilibrium constant of the adsorption process, $f$ is a factor of energetic in homogeneity in the surface, $\theta$ is the coverage rate while $K$ is related to $K_{a d s}\left(K_{a d s}=K^{1 / y}\right)$

Figures 3B-D give the representations of the tested adsorption isotherms. The parameters of all tested isotherms are listed in Table 1.

Table 1: Isotherms parameters for various temperatures.

\begin{tabular}{lllll}
\hline Isotherm & & & & \\
model & $T(K)$ & $R^{2}$ & Slope & Intercept \\
\hline Langmuir & 298 & 0.9995 & 1.0595 & 0.0225 \\
& 303 & 0.9990 & 1.0829 & 0.0274 \\
& 308 & 0.9987 & 1.1255 & 0.0304 \\
& 313 & 0.9987 & 1.1482 & 0.0321 \\
& 318 & 0.9983 & 1.1943 & 0.0365 \\
\hline Temkin & 298 & 0.9967 & 0.1145 & 0.9012 \\
& 303 & 0.9844 & 0.1106 & 0.8753 \\
& 308 & 0.9707 & 0.1024 & 0.8396 \\
& 313 & 0.9888 & 0.1143 & 0.8221 \\
& 318 & 0.9842 & 0.1129 & 0.7872 \\
\hline El-Awady & 298 & 0.9597 & 0.3923 & 1.0072 \\
& 303 & 0.9373 & 0.3369 & 0.8822 \\
& 308 & 0.9302 & 0.2722 & 0.7404 \\
& 313 & 0.9620 & 0.2782 & 0.6819 \\
& 318 & 0.9615 & 0.2502 & 0.5800 \\
\hline
\end{tabular}

The choice of the best adsorption isotherm was based on the values of the correlation coefficients $\left(R_{2}\right)$. As it can be seen in Table 1, by far the results are better fitted by Langmuir adsorption isotherm as the linear correlation coefficients $\left(R_{2}\right)$ are almost equal to 1 and the slopes are close to 1 . These results confirm that the adsorption of 7-ETBT on aluminum surface better follows Langmuir adsorption isotherm.

In order to evaluate the strength of the interactions between the inhibitor molecules and the metal surface, the values of adsorption equilibrium constant $K_{a d s}$ were calculated using the intercepts of the straight lines on $\frac{C_{i n h}}{\theta}$-axis. The calculated adsorption equilibrium constant was related to the standard free adsorption enthalpy by the following equation (Keles et al., 2008):

$$
\Delta G_{a d s}^{0}=-R T \ln \left(55.5 K_{a d s}\right)
$$

In the above equation (Villamil et al., 1999), 55.5 is the concentration of water in mol. $\mathrm{L}^{-1}$, $\mathrm{T}$ is the absolute temperature while $\mathrm{R}$ is the perfect gas constant. The values of $\frac{C_{i n h}}{\theta}$ and the other adsorption thermodynamic functions are summarized in Table 2.

\begin{tabular}{|l|l|l|l|l|}
\hline \multicolumn{5}{|c|}{ Table 2: Adsorption thermodynamic functions. } \\
\cline { 1 - 3 }$T(K)$ & $K_{\text {ads }}\left(\mathrm{M}^{-1}\right)$ & $\Delta G_{\text {ads }}^{0}\left(\mathrm{KJmol}^{-1}\right)$ & \multirow{2}{*}{$\Delta H_{\text {ads }}^{0}\left(\mathrm{KJmol}^{-1}\right)$} & $\Delta S_{\text {ads }}^{0}\left(\mathrm{Jmol}^{-1} \mathrm{~K}^{-1}\right)$ \\
\hline 298 & 44444.44 & -36.45 & & \\
\hline 303 & 36496.35 & -36.56 & \multirow{3}{*}{-17.636} & \multirow{2}{*}{62.80} \\
\hline 308 & 32894.74 & -36.90 & & \\
\hline 313 & 31152.65 & -37.36 & & \\
\hline 318 & 27397.26 & -37.62 & & \\
\hline
\end{tabular}

In our work, the computed values of $\Delta G_{\text {ads }}^{0}$ for 7-ETBT were ranging from -36.45 to $-37.62 \mathrm{kJmol}^{-1}{ }^{-1 s}$ which indicated that the adsorption of 7-ETBT on the aluminum surface may involve physical as well as chemical adsorption (Khaled and Hackerman, 2004; Quraishi et al., 2010) and the decrease in values of $K_{a d s}$ with increasing temperature suggested that the desorption process enhances with the increase in temperature (Quraishi et al., 2010). The large negative values of $\Delta G_{a d s}^{0}$ reveal that the adsorption process takes place spontaneously and the adsorbed layer on the surface of aluminum is highly stable (Scendo and Hepel, 2008).

The standard adsorption enthalpy change $\Delta H_{a d s}^{0}$ and the standard adsorption entropy change $\Delta S_{a d s}^{0}$ are correlated with standard Gibbs free energy by Equation 9:

$\Delta G_{a d s}^{0}=\Delta H_{a d s}^{0}-T \Delta S_{a d s}^{0}$

Figure $4 \mathrm{~A}$ gives the plots of $\Delta G_{a d s}^{0}$ versus the temperature. The values of $\Delta H_{a d s}^{0}$ and $\Delta S_{a d s}^{0}$ obtained by linear regression are listed in Table 3. $\Delta H_{\text {als }}^{0}$ is negative, showing an exothermic process. According to the literature (Durnie et al., 1999) an exothermic process means either physisorption or chemisorption. Therefore this result confirms that the process of adsorption is both physisorption and chemisorption (Diki et al., 2018b). $\Delta S_{a d s}^{0}$ is positive, meaning that disorder increases during the adsorption process. This situation can be explained by the desorption of water molecules replaced by the inhibitor.

\subsubsection{Effect of temperature and activation parameters of the corrosion process}

The apparent activation energy $\left(E_{a}\right)$ can be calculated by using Arrhenius equation (Equation 10):

$\log W=\log A-\frac{E_{a}}{2.303 R T}$

Where, $\mathrm{W}$ is the corrosion rate, $\mathrm{A}$ is the Arrhenius preexponential constant, $\mathrm{R}$ is the molar gas constant and $\mathrm{T}$ is the absolute temperature. A plot of $\log W$ versus $1 / \mathrm{T}$ yields 

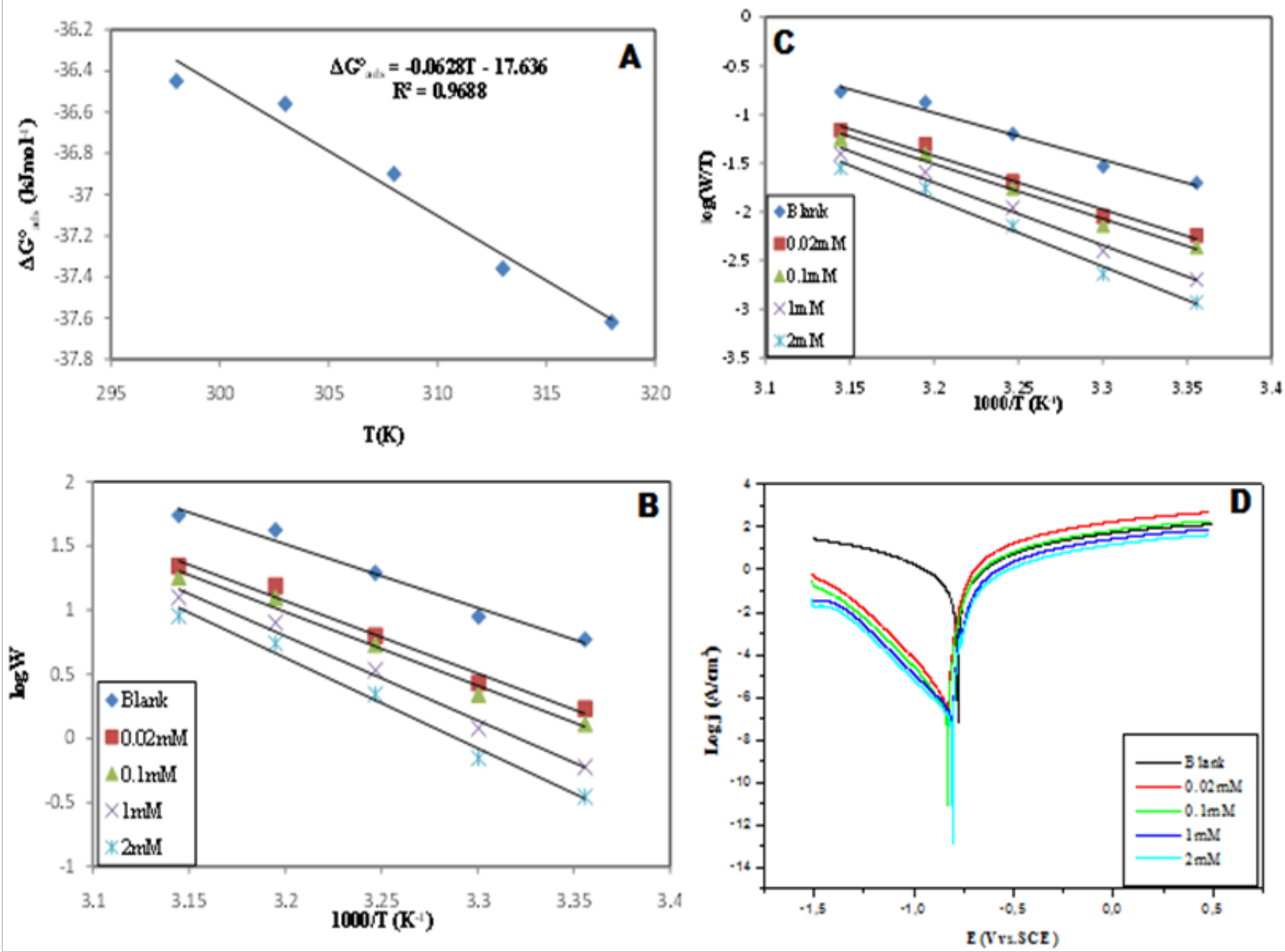

Figure 4: A) Free adsorption enthalpy $\Delta G_{\text {ads }}^{0}$ versus temperature, B) Arrhenius plots for aluminum corrosion in $1 \mathrm{M} \mathrm{HCl}$, C) Transition state plots of the adsorption behavior of 7-ETBT and D) Potentiodynamic polarization curves obtained for aluminum at $298 \mathrm{~K}$ in $1 \mathrm{M} \mathrm{HCl}$ in various concentrations of 7 -ETBT.

a straight line (Figure $4 \mathrm{~B}$ ) with $-E_{a} / 2.303 R$
$\log \mathrm{A}$ as intercept. The other activation parameters for the corrosion process were calculated from the Arrhenius equation:

$$
\log \left(\frac{W}{T}\right)=\left[\log \left(\frac{R}{\aleph h}\right)+\frac{\Delta S_{a}^{*}}{2.303 R}\right]-\frac{\Delta H_{a}^{*}}{2.303 R T}
$$

Where, $\Delta S^{*}$ is the change in apparent activation entropy, $\Delta H^{*}$. is the change in apparent activation enthalpy, $R$ is the perfect gas constant, $\boldsymbol{N}$ is the Avogadro number and $\mathrm{h}$ is the Planck's constant.

Figure $4 \mathrm{C}$ gives the plot of $\log \left(\frac{W}{T}\right)$ versus $1 / \mathrm{T}$. The slope $-\frac{A H_{a}^{*}}{2.303 R T}$ and the intercept $\left[\log \left(\frac{R}{\kappa h}\right)+\frac{n S_{a}^{*}}{2.303 R}\right]$ straight line lead to the values of activation enthalpy change and activation entropy change (Table 3 ).

From Table 3, it seems that $E_{a}$ and $\Delta H_{a}^{*}$ varied in the same manner increasing with the concentration, probably due to the thermodynamic relation between them $\left(\Delta H_{a}^{*}=E_{a}-R T\right)$. It can be seen that the values of $E_{a}$ are higher in the inhibited solutions than those in uninhibited solutions. On the other hand, the higher values of
Table 3: Calculated activation energy, activation enthalpy change and activation entropy change.

\begin{tabular}{llll}
\hline System & $E_{a}(\mathrm{kJmol}-1)$ & $\Delta H_{a}^{*}\left(\mathrm{kJmol}^{-1}\right)$ & $\Delta S_{a}^{*}\left(\mathrm{Jmol}^{-1} \mathrm{~K}^{-1}\right)$ \\
\hline Blank & 94.77 & 92.09 & 78.73 \\
$0.02 \mathrm{mM}$ & 108.11 & 107.97 & 112.91 \\
$0.1 \mathrm{mM}$ & 110.23 & 110.09 & 118.07 \\
$1 \mathrm{mM}$ & 125.94 & 125.78 & 164.65 \\
$2 \mathrm{mM}$ & 134.90 & 134.72 & 190.03 \\
\hline
\end{tabular}

$E_{a}$ in the presence of inhibitor compared to that in its absence and the decrease of the inhibition efficiency (IE) with the increase in temperature can be interpreted as an indication of predominant physical adsorption process (Chaubey et al., 2017; DIKI et al., 2018a; Umoren, 2008). Moreover, the positive signs of $\Delta H^{*}$ reflected the endothermic effect of the aluminum dissolution process. The value of $\Delta S^{*}$ is higher for the inhibited solution than that for the uninhibited solution. This phenomenon suggested that a randomness decrease occurred from reactants to the activated complex. This might be the result of the adsorption of organic inhibitor molecules from the acidic solution which could be regarded as a quasi-substitution 
process between the organic compound in the aqueous phase and water molecules at the metal surface (Afia et al., 2012).

\subsection{Potentiodynamic polarization measurements}

For the purpose of detecting the anodic, cathodic or mixed nature of the studied inhibitor, potentiodynamic polarization measurements have been carried out. Polarization curves for aluminum in $1 \mathrm{M} \mathrm{HCl}$ without and with 7-ETBT at different concentrations are shown in Figure 4D. The anodic and cathodic current-potential curves are extrapolated up to their intersection at the point where corrosion current density $\left(j_{\text {corr }}\right)$ and corrosion potential $\left(E_{c o r r}\right)$ are obtained. The effect of increasing concentration of 7-ETBT on the anodic and cathodic polarization curves of $\mathrm{Al}$ in $1 \mathrm{M} \mathrm{HCl}$ solution at $298 \mathrm{~K}$ is also presented in Figure 4D. In all cases, addition of the 7-ETBT induced a marked decrease in the cathodic current density and a slight decrease for the two highest concentrations ( 1 and $2 \mathrm{mM}$ ) of 7-ETBT in anodic current density. As a result, the tested inhibitor slightly affected the dissolution of the metal and greatly affected the hydrogen evolution reaction. In our case, cathodic Tafel slopes are comparable to parallel straight lines. This suggests that the hydrogen evolution reaction is under activation controlled (Bentiss, 2006). Therefore, the addition of the inhibitor to the corrosive solution does not modify the mechanism of the proton reduction process. The sharp decrease in the cathodic process can be due to the covering of the metal surface with monolayer of the inhibitor molecules due to the adsorbed inhibitor molecules (Khaled and AlQahtani, 2009). The electrochemical parameters $E_{\text {corr }} j_{\text {corr }}$ IE, anodic and cathodic Tafel slopes $\left(b_{a r} b_{c}\right)$ obtained from the polarization measurements are displayed in Table 4 .

From Table 4, anodic and cathodic Tafel slopes are almost constant in the presence of 7-ETBT, meaning that the inhibiting action of this molecule occurred by simple blocking of the available surface area (Azim et al., 1974; Quraishi et al., 2010). Furthermore, it is clear that the corrosion current density $\left(\mathrm{j}_{\text {corr }}\right)$ values decrease from 201.06

Table 4: Electrochemical kinetic parameters obtained from potentiodynamic polarization curves in $1 \mathrm{M} \mathrm{HCl}$ in different concentrations of 7-ETBT at $298 \mathrm{~K}$.

\begin{tabular}{llllll}
\hline & $\begin{array}{l}E_{\text {corr }} \\
(m V v s .\end{array}$ & $j_{\text {corr }}$ & $b_{a}(m V /$ & $-b_{c}(m V /$ & \\
$C_{\text {inh }}(m M)$ & $S C E)$ & $\left(\mu A / m^{2}\right)$ & $d e c)$ & $d e c)$ & $I E(\%)$ \\
\hline 0 (Blank) & -783.53 & 201.06 & 154.85 & 115.32 & - \\
0.02 & -869.42 & 62.30 & 158.13 & 204.02 & 69.01 \\
0.1 & -870.30 & 47.83 & 157.52 & 199.60 & 76.21 \\
1 & -872.61 & 26.50 & 171.93 & 198.68 & 86.81 \\
2 & -872.68 & 18.00 & 169.80 & 202.90 & 91.05 \\
\hline
\end{tabular}

$\mu \mathrm{A} / \mathrm{cm}^{2}$ to $18.00 \mu \mathrm{A} / \mathrm{cm}^{2}$ with the addition of various concentrations of 7-ETBT. When the current density $\left(j_{\text {corr }}\right)$ decreases, the inhibition efficiency (IE) increases from $69.01 \%$ to $91.05 \%$. It is known that when the value of $E_{\text {corr }}$ shifts beyond $85 \mathrm{mV}$, the chemical compound can be designated as an anodic or a cathodic-type inhibitor. As $E_{\text {corr }}$ values cathodically shifted in the presence of 7-ETBT with the maximum displacement about $89.15 \mathrm{mV}$ vs. SCE which is more than $85 \mathrm{mV}$. This result suggests that 7-ETBT is a cathodic-type inhibitor (Ansari and Quraishi, 2015; Satapathy et al., 2009). From the potentiodynamic polarization data, one can see that the inhibition efficiencies obtained with the potentiodynamic polarization method are in agreement with those obtained from the mass loss measurements over the inhibitor concentration range studied. These results suggest that 7-ETBT can be considered as an efficient metal inhibitor in acidic aqueous media.

\subsection{Surface characterization}

In order to characterize the metal surface behavior in contact with chloride acid solution in the absence and presence of the studied inhibitor, a surface analysis was carried out using a scanning electron microscope, immediately after the corrosion tests. The aluminum samples in $1 \mathrm{M} \mathrm{HCl}$ solution with and without optimal concentration of the 7-ETBT were subjected to analysis. SEM images are shown in Figure $5 \mathrm{~A}-\mathrm{C}$.

As displayed, without inhibitor (Figure 5C), the metal surface is subject to corrosion. Severe damage, clear pits and cavities are observed on the surface of aluminum in the absence of inhibitor compared with polished bare metal before immersion (Figure $5 \mathrm{~A})$. In the presence of 7-ETBT (Figure 5B), the metal surface is not available confirming that the metal surface is fully covered with the inhibitor molecules and a protective inhibitor film was formed. These results suggest the inhibitor effect of 7-ETBT.

\subsection{Quantum calculations}

The electronic parameters describing inhibitor molecules can be assessed at two different levels: the global level and the local one.

\subsubsection{Global parameters}

Recently, DFT has become a very powerful technique to probe the inhibitor/surface interaction and to analyze experimental data by providing insights into the chemical reactivity and selectivity (Patel et al., 2014; Rodriguez-Valdez et al., 2005). So, the molecular descriptors namely the highest occupied molecular orbital energy $\left(\mathrm{E}_{\mathrm{HOMO}}\right)$, the 

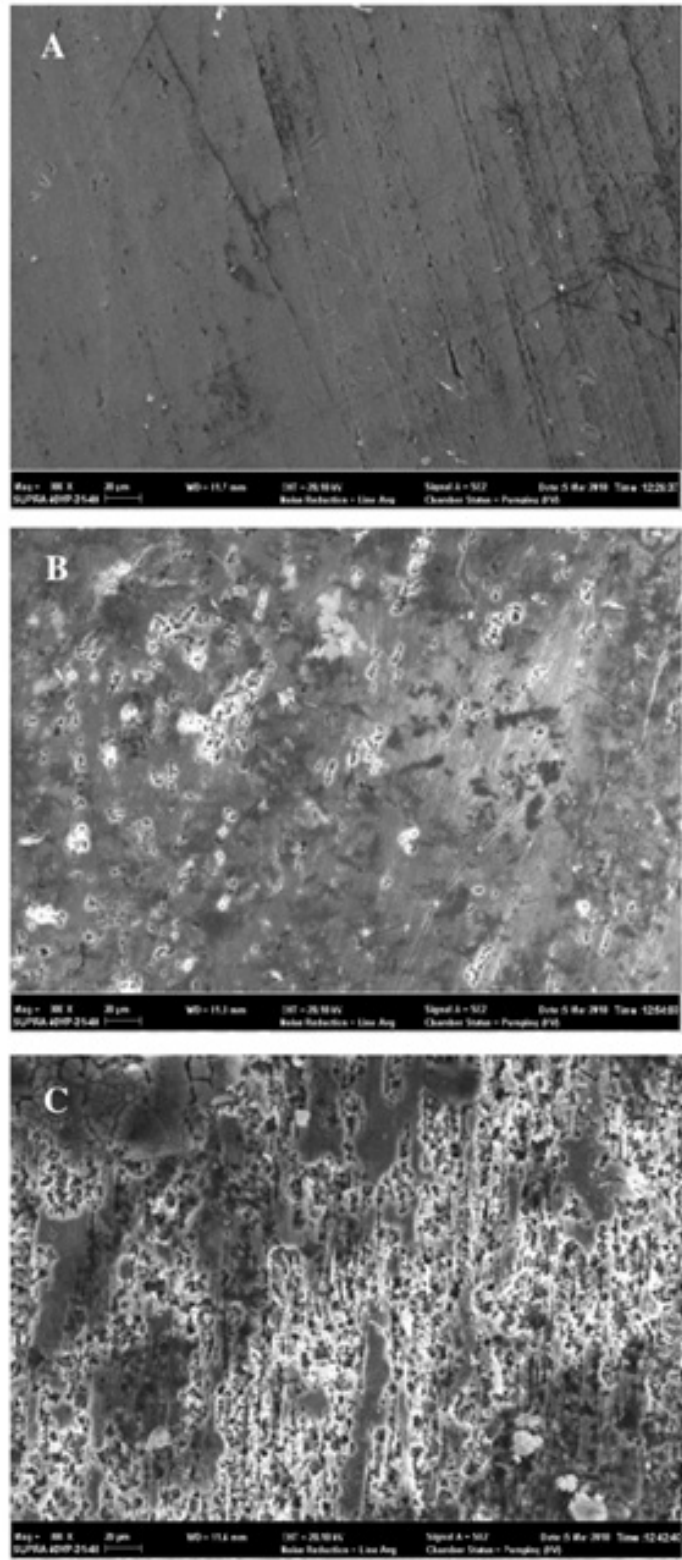
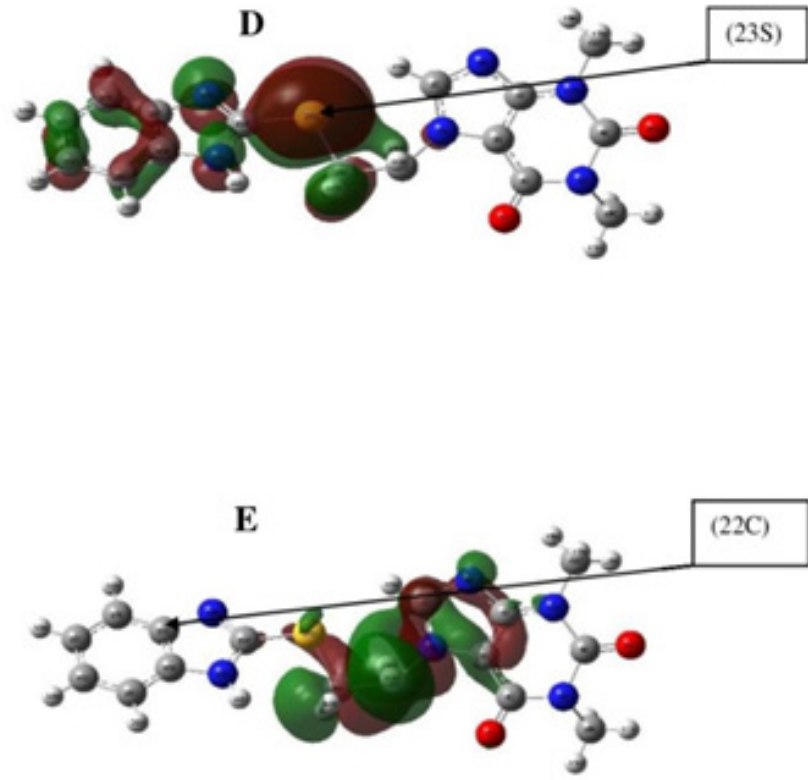

Figure 5: SEM images of aluminum (A) Before immersion in $1 \mathrm{M} \mathrm{HCl}$, and after $1 \mathrm{~h}$ of immersion in $1 \mathrm{M} \mathrm{HCl} \mathrm{solution} \mathrm{(C)} \mathrm{without} \mathrm{and}$ (B) with 7-ETBT (2 mM). (D) HOMO, (E) LUMO of 7-ETBT by B3LYP/6-31G (d, p).

lowest unoccupied molecular orbital energy (ELUMO), the energy gap ( $\left.\triangle \mathrm{E}=\mathrm{E}_{\mathrm{LUMO}}-\mathrm{E}_{\mathrm{HOMO}}\right)$ and the dipole moment $(\mu)$ were calculated. The reactivity descriptors, including ionization energy (I), electron affinity (A), electronegativity $(\chi)$, hardness $(\eta)$, softness $(S)$, the fraction $(\Delta \mathrm{N})$ of electrons transferred and the electrophilicity index $(\omega)$ were also calculated. According to Koopman's theorem (Koopmans, 1934), the ionization potential I can be approximated as the negative of the highest occupied molecular orbital (HOMO) energy (Equation 12):

$\mathrm{I}=-\mathrm{E}_{\mathrm{HOMO}}$

The negative of the lowest unoccupied molecular orbital (LUMO) energy is related to the electron affinity $A$ and is obtained from Equation 13:

$\boldsymbol{A}=-\boldsymbol{E}_{\text {LUMO }}$
The electronegativity (Parr et al., 1978) is obtained using the ionization energy I and the electron affinity A by Equation 14:

$\chi=\frac{I+A}{2}$

The hardness which is the reciprocal of the electronegativity was obtained by Equation 15:

$\eta=\frac{I-A}{2}$

When the organic molecule is in contact with the metal, electrons flow from the system with lower electronegativity to that of higher electronegativity until the chemical potential becomes equal. The fraction of electrons transferred, $\Delta N$, was estimated according to Equation 16 (Kokalj and Kovacevic, 2011):

$$
\Delta \boldsymbol{N}=\frac{\Phi_{A l}-\chi_{i n h}}{2\left(\eta_{A l}+\eta_{i n h}\right)}
$$


The values of experimental work function $\Phi_{A l}=4.28 \mathrm{eV}$ (Michaelson, 1977) and hardness $\eta_{A l}=0$ (Sastri and Perumareddi, 1997) (since for bulk metallic atoms $I=A$ ) were considered to calculate $\Delta N$.

The global electrophilicity index, introduced by Parr (Parr et al., 1999) is given by Equation 17:

$\omega=\frac{\chi^{2}}{2 \eta}$

The quantum chemistry parameters of 7-ETBT were calculated and listed in Table 5.

According to the frontier molecular orbital (FMO) theory, of chemical reactivity, transition of electron is due to interaction between highest occupied molecular

Table 5: Molecular and global reactivity descriptors of 7-ETBT.

\begin{tabular}{llll}
\hline Descriptor & Value & Descriptor & Value \\
\hline $\mathrm{E}_{\text {HOMO }}(\mathrm{eV})$ & -5.7950 & $\mathrm{I}(\mathrm{eV})$ & 5.7950 \\
$\mathrm{E}_{\mathrm{LUMO}}(\mathrm{eV})$ & -1.1778 & $\mathrm{~A}(\mathrm{eV})$ & 1.1778 \\
$\Delta \mathrm{E}(\mathrm{eV})$ & 4.6172 & $\mu($ Debye $)$ & 5.5740 \\
$\Delta \mathrm{N}$ & 0.1719 & $\eta(\mathrm{eV})$ & 2.3086 \\
$\mathrm{~S}(\mathrm{eV})^{-1}$ & 0.4332 & $\omega$ & 2.6325 \\
$\Delta(\mathrm{eV})$ & 3.4864 & $\mathrm{TE}(\mathrm{a} . \mathrm{u})$ & -1496.5743 \\
\hline
\end{tabular}

orbital (HOMO) and lowest unoccupied molecular orbital (LUMO) of reacting species (Lukovits et al., 1997; Musa et al., 2010) are very important in defining the reactivity of a given compound. $\mathrm{E}_{\mathrm{HOMO}}$ (Popova et al., 2003) is often associated with the electron donating ability of a molecule. High $\mathrm{E}_{\mathrm{HOMO}}$ values indicate that the molecule has a tendency to donate electrons to appropriate acceptor molecules with low energy empty molecular orbital. Increasing values of the $\mathrm{E}_{\mathrm{HOMO}}$ facilitate adsorption (and therefore inhibition) by influencing the transport process through the adsorbed layer (Özcan and Dehri, 2004). On the other hand, the LUMO energy (Popova et al., 2003), indicates the ability of the molecule to accept electrons. The lower the value of $\mathrm{E}_{\mathrm{HOMO}}$, the more probable it is that the molecule accepts electrons. In this work, the higher value of $\mathrm{E}_{\mathrm{HOMO}}(-5.7950 \mathrm{eV})$ and the lower value of $\mathrm{E}_{\mathrm{LUMO}}$ $(-1.1778 \mathrm{eV})$ could involve charge sharing or charge transfer from the inhibiting molecule to the vacant p-orbital of aluminum (Lebrini et al., 2005). That could explain good inhibition efficiency of 7-ETBT. In the same way, low values of the energy gap ( $\triangle \mathrm{E}=\mathrm{E}_{\mathrm{LUMO}}-\mathrm{E}_{\mathrm{HOMO}}$ ) lead to high inhibition efficiencies because (F., 2003) the energy to remove an electron from the last occupied orbital will be low. Literature (Ouédraogo et al., 2018) revealed that excellent corrosion inhibitors are organic compounds which not only offer electrons to unoccupied orbital of a metal but also accept free electrons from the metal. A molecule with a low energy gap (Obot et al., 2009) is more polarizable and is generally associated with high chemical reactivity and is considered as a soft molecule. The value of $\Delta E(4.6172 \mathrm{eV})$ is low compared with that of many molecules in the literature, suggesting good inhibition efficiency as obtained from mass loss and Tafel polarization methods.

Another important electronic parameter calculated is the dipole moment $(\mu)$ that results from non-uniform distribution of charges on atoms in the molecule. Several authors point out that low values of dipole moment (Khalil, 2003) promote accumulation of the inhibitor molecules in the surface layer and therefore higher inhibition efficiency. However, many papers indicate that inhibition efficiency increases with rising values of dipole moment. On the other hand, survey of the literature (Bereket et al., 2002; Khaled et al., 2005) reveals that several irregularities appeared in case of correlation of dipole moment with inhibitor efficiency. So, in general (Gece, 2008), there is no significant relationship between dipole moment values and inhibition efficiencies.

Absolute hardness and softness are important parameters to measure the molecular stability and reactivity of a molecule. The chemical hardness fundamentally represents the resistance towards the deformation or polarization of the electron cloud of atoms, ions or molecules under small perturbation of chemical reaction. A hard molecule has a large energy gap and a soft molecule has a small energy gap (Gece and Bilgic, 2009). In our work, the studied molecule has a low hardness value $(2.3086 \mathrm{eV})$ and a high value of softness $(0.4332 \mathrm{eV})^{-1}$ when compared (Udhayakala and Rajendiran, 2015; Udhayakala et al., 2012) with molecules in the literature.

The ionization potential (I) and the electronic affinity (A) are respectively $(5.7950 \mathrm{eV})$ and $(1.1778 \mathrm{eV})$. This low value of (I) and the high value of electron affinity indicate the capacity of the molecule both to donate and accept electron. The electronegativity $(\chi)$ indicates the capacity of a system to attract electrons. In our work the low value of the electronegativity of the studied molecule $(\chi=3.4864 \mathrm{eV})$ when compared to that of aluminum $\left(\Phi_{A l}=4.28 \mathrm{eV}\right)$ shows that aluminum has the better attraction capacity. Then the low value of hardness $(2.3086 \mathrm{eV})$ confirms the relatively higher value of the fraction of electrons transferred $(\Delta N=0.1719)$ indicating a possible motion of electrons from the inhibitor to the metal. The electrophilicity index measures the propensity of chemical species to accept electrons; a high value of electrophilicity index describes a good electrophile while a small value of electrophilicity index describes a good nucleophile. In this work the obtained value $(\omega=2.6325 \mathrm{eV})$ shows the good capacity of 7-ETBT to accept electrons. The HOMO and LUMO densities of 7-ETBT are shown in Figure 5D and E.

The analysis of Figure 5D\&E show that the density HOMO (see Figure 5D) and LUMO (see Figure 5E) for this compound are distributed only around the benzimida- 
zole cycle for HOMO and only around theophylline cycle for LUMO respectively, suggesting that these regions are probably the active areas where transfers of electrons could occur (from 7-ETBT to aluminum or vice-versa).

\subsubsection{Local parameters}

The local selectivity of a corrosion inhibitor (Fuentealba et al., 2000) is generally assessed using Fukui functions which enable us to distinguish each part of the studied compound on the basis of its chemical behavior due to different substituent functional groups. The Fukui function is defined as the derivative of the electronic density $\mathrm{Q}(\mathrm{r})$ with respect to the number $\mathrm{N}$ of electrons:

$f(r)=\left(\frac{\partial \rho(r)}{\partial N}\right)_{v(r)}$

The condensed Fukui functions provide information about atoms in a molecule that have a tendency to either donate (nucleophilic character) or accept (electrophilic character) an electron or a pair of electrons (Kouakou et al., 2016). The nucleophilic and electrophilic Fukui function for an atom k (Eddy et al., 2010) can be computed using a finite difference approximation as seen in Equation 19-20 respectively:

$f_{k}^{+}=\left[q_{k}(N+1)-q_{k}(N)\right]$ for nucleophilic attack

$f_{k}^{-}=\left[q_{k 1}(N)-q_{k}(N-1)\right]$ for electrophilic attack

Where,

$q_{k}(\mathrm{~N}+1), q_{k}(\mathrm{~N})$ and $q_{k}(\mathrm{~N}-1)$ are the charges of the atoms on the systems with $(\mathrm{N}+1), \mathrm{N}$ and $\mathrm{N}-1$ electrons respectively.

Recently, it has been reported (Yeo et al., 2017) that a new descriptor has been introduced (Morell et al., $2005,2006)$ which allows the determination of individual sites within the molecule with particular behaviors. A mathematical analysis reveals that dual descriptor is a more accurate tool than nucleophilic and electrophilic Fukui functions (Martinez-Araya, 2015). This descriptor is defined through Equation 21:

$\Delta f(r)=\left(\frac{\partial f(r)}{\partial N}\right)_{v(r)}$

The condensed form (Morell et al., 2006) of the dual descriptor is given by Equation 22:

$\Delta f_{k}(r)=f_{k}^{+}-f_{k}^{-}$

When, $\Delta f_{k}(r)>0$, the process is driven by a nucleophilic attack and atom $k$ acts as an electrophile; conversely, when, $\Delta \mathrm{f}_{k}(r)<0$ the process is driven by an electrophilic attack on atom $\mathrm{k}$ acts as a nucleophile. The dual descriptor $\Delta \mathrm{f}_{k}(r)$ is defined within the range $[-1 ; 1]$, what really facilitates interpretation (Martinez-Araya, 2015). The computed Mulliken atomic charges, Fukui functions and dual descriptor by DFT at the B3YLP/6-31G $(\mathrm{d}, \mathrm{p})$ level are gathered in Table 6.

From shaded rows in Table 6, one can see that (22 C) with the maximum value of $\mathrm{fk}+$ and positive value of $\Delta f_{k}$ $(r)$ is the preferred nucleophilic attack site while (23 S)

Table 6: Calculated Mulliken atomic charges, Fukui functions and dual descriptor by DFT B3YLP/6-31 G (d, p).

\begin{tabular}{lrrrrrr}
\hline Atom & $q_{k}(N+1)$ & $q_{k}(N)$ & $q_{k}(N-1)$ & \multicolumn{1}{l}{$f_{k}^{+}$} & \multicolumn{1}{l}{$f_{k}^{-}$} & \multicolumn{1}{l}{$\Delta f$} \\
\hline 1 C & 0.457 & 0.471 & 0.532 & -0.014 & -0.061 & 0.047 \\
2 C & 0.229 & 0.216 & 0.220 & 0.013 & -0.004 & 0.017 \\
3 C & 0.293 & 0.228 & 0.291 & 0.065 & -0.063 & 0.128 \\
5 N & -0.553 & -0.544 & -0.605 & -0.009 & 0.061 & -0.070 \\
6 N & -0.520 & -0.527 & -0.540 & 0.007 & 0.013 & -0.006 \\
7 C & 0.619 & 0.633 & 0.669 & -0.014 & -0.036 & 0.022 \\
8 O & -0.553 & -0.545 & -0.517 & -0.008 & -0.028 & 0.020 \\
9 N & -0.588 & -0.590 & -0.593 & 0.002 & 0.003 & -0.001 \\
10 C & 0.779 & 0.788 & 0.799 & -0.009 & -0.011 & 0.002 \\
11 C & -0.162 & -0.170 & -0.181 & 0.008 & 0.011 & -0.003 \\
15 O & -0.559 & -0.527 & -0.491 & -0.032 & -0.036 & 0.004 \\
16 N & -0.601 & -0.608 & -0.619 & 0.007 & 0.011 & -0.004 \\
17 C & -0.171 & -0.177 & -0.197 & 0.006 & 0.020 & -0.014 \\
21 C & -0.092 & -0.028 & -0.099 & -0.064 & 0.071 & -0.135 \\
22 C & -0.217 & -0.406 & -0.202 & $\mathbf{0 . 1 8 9}$ & -0.204 & 0.393 \\
23 S & -0.307 & 0.471 & 0.342 & -0.778 & $\mathbf{0 . 1 2 9}$ & -0.907 \\
28 C & 0.237 & 0.316 & 0.308 & -0.079 & 0.008 & -0.087 \\
29 C & 0.317 & 0.228 & 0.357 & 0.089 & -0.129 & 0.218 \\
31 N & -0.592 & -0.523 & -0.591 & -0.069 & 0.068 & -0.137 \\
32 N & -0.613 & -0.632 & -0.623 & 0.019 & -0.009 & 0.028 \\
33 C & 0.315 & 0.316 & 0.301 & -0.001 & 0.015 & -0.016 \\
34 C & -0.122 & -0.104 & -0.084 & -0.018 & -0.020 & 0.002 \\
35 C & -0.133 & -0.107 & -0.081 & -0.026 & -0.026 & 0.000 \\
36 C & -0.105 & -0.100 & -0.091 & -0.005 & -0.009 & 0.004 \\
37 C & -0.115 & -0.104 & -0.090 & -0.011 & -0.014 & 0.03 \\
\hline & & & & & &
\end{tabular}

with the maximum value of fk- and negative value of $\Delta f_{k}$ $(r)$ is the preferred electrophilic attack site. These results are confirmed by the great electron cloud on (23S) (Figure 5D) and the absence of electron density on (22 C) (Figure 5E).

\section{INHIBITION MECHANISM}

Inhibition of aluminum by 7-ETBT in hydrochloric acid solutions might be related to the molecular structure of the inhibitor which possesses several heteroatoms $(\mathrm{N}$, $\mathrm{S}, \mathrm{O})$ and delocalized $\pi$-electrons of heterocyclic rings. In acid aqueous solution, the studied inhibitor exists as protonated species. The molecules of 7-ETBT could be protonated since they contain heteroatoms:

$7-\mathrm{ETBT}+\mathrm{xH} \mathrm{H}^{+} \leftrightarrow\left[7-\mathrm{ETBTH}_{\mathrm{x}}\right]^{\mathrm{x}}$

Due to their specific adsorption and their small degree of hydratation, chloride ions $\left(\mathrm{Cl}^{-}\right)$from $\mathrm{HCl}$ firstly adsorbed on the positively charged metal surface $\left(A l^{(3+)}\right)$ 


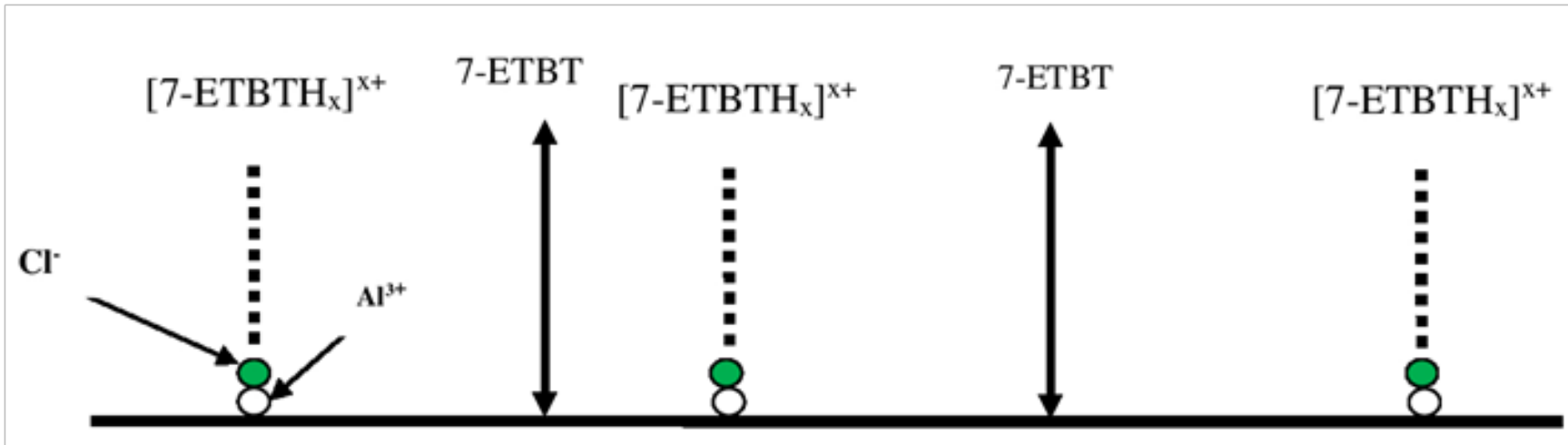

Aluminum

Figure 6: Schematic mechanism of Al corrosion inhibition in $1 \mathrm{M} \mathrm{HCl}$ by $7-\mathrm{ETBT}$.

ions cover the surface of the metal) and create an excess negative charge along the metal surface towards the solution side, what leads to the adsorption of the protonated form of the inhibitor (Luo et al., 1998).

Therefore, a protective layer due to the electrostatic interaction between the charged species from the inhibitor and the chloride ions is formed (physisorption). The positive sign of fraction of electron transferred shows that electrons can be transferred from the inhibitor species to the aluminum and that positive charged species have a strong tendency to accept electron from porbital of the aluminum to form a bond, what could explain the existence of chemisorption. So, a probable schematic mechanism (Fig. 6) can be proposed where the black arrows point out chemisorption, whereas the broken lines show the electrostatic interaction between the chloride ions and the cationic form of the inhibitor (physisorption).

\section{CONCLUSION}

The results of this study can be concluded as follows:

- The inhibition efficiency is temperature and concentration dependent;

- The studied molecule adsorbs on aluminum according to the Langmuir isotherm;

- The values of adsorption thermodynamic functions and that of the activation energy suggest both physisorption and chemisorption with a predominant physisorption;

- Tafel polarization data reveal that 7-ETBT is a cathodic-type inhibitor;

- The SEM images confirm the formation of a protective layer on the metal surface;

- The quantum descriptors confirm the good inhibition efficiency of 7-ETBT;

- Theoretical results are consistent with the experimental data;
- 7-ETBT is an effective inhibitor for aluminum corrosion in $1 \mathrm{M} \mathrm{HCl}$;

- 7-ETBT could be an efficient metal corrosion inhibitor and could be used for industrial equipment protection such as petroleum pipeline where fluid leakage is prevalent in order to avoid an environmental pollution.

\section{ACKNOWLEDGEMENTS}

The present experiments were carried out at Physical Chemistry Laboratory and Laboratory of Organic Chemistry and Natural Substances of Felix Houphouet-Boigny University, Abidjan, Côte d'Ivoire.

\section{REFERENCES}

1. Afia L, Salghi R, Zarrouk A, Zarrok H, et al., (2012). Inhibitive action of argan press cake extract on the corrosion of steel in acidic media. Port. Electrochim. Acta. 30:267-279.

2. Ajmal M, Mideen AS, Quraishi MA (1994). 2-hydrazino6-methyl-benzothiazole as an effective inhibitor for the corrosion of mild steel in acidic solutions. Corros. Sci. 36:79-84.

3. Ansari KR, Quraishi MA (2015). Effect of three component (aniline-formaldehyde and piperazine) polymer on mild steel corrosion in hydrochloric acid medium. J. Assoc. Arab Univ. Basic Appl. Sci. 18:12-18.

4. Azim AAA, Shalaby LA, Abbas H (1974). Mechanism of the corrosion inhibition of $\mathrm{Zn}$ Anode in $\mathrm{NaOH}$ by gelatine and some inorganic anions. Corros. Sci. 14:21-24.

5. Badr GE (2009). The role of some thiosemicarbazide derivatives as corrosion inhibitors for C-steel in acidic media. Corros. Sci. 51:2529-2536.

6. Bentiss F (2006). Hétérocycles pentaatomiques : Synthèses organiques, études des propriétés inhibitrices de la corrosion et des propriétés complexantes. H D R Université de Lille 1, France. 233 P.

7. Bereket G, Hur E, Ogretir C (2002). Quantum chemical studies on some imidazole derivatives as corrosion inhibitors for iron in acidic medium. J. Mol. Struct.: Theochem. 578:79-88.

8. Berkowitz M, Ghosh SK, Parr RG (1985). On the concept of local hardness in chemistry. J. Am. Chem. Soc. 107:6811-6814. 
9. Chaubey N, Savita Singh VK, Quraishi MA (2017). Corrosion inhibition performance of different bark extracts on aluminium in alkaline solution. J. Assoc. Arab Univ. Basic Appl. Sci. 22:38-44.

10. Cruz J, Martinez R, Genesca J, Garcia-Ochoa E (2004). Experimental and theoretical study of 1-(2-ethylamine-2-methylimidazoline as an inhibitor of carbon steel corrosion in acid media. J. Electroanal. Chem. 566:111-121.

11. Cruz J, Pandiyan T, Chemistry EG.-O.J.o.E (2005). A new inhibitor for mild carbon steel: electrochemical and DFT studies. J. Electroanal. Chem. 583:8-16.

12. Desai MN, Desai MB, Shah CB, Desai SM (1986). Schiff bases as corrosion inhibitors for mild steel in hydrochloric acid solutions. Corros. Sci. 26:827-837.

13. Desimone MP, Gordillo G, Simison SN (2011). The effect of temperature and concentration on the corrosion inhibition mechanism of an amphiphilic amido-amine in $\mathrm{CO} 2$ saturated solution. Corro. Sci. 53:4033-4043.

14. Diki NYS, Bohoussou KV, Kone MG-R., Ouedraogo A, Trokourey A (2018a). Cefadroxil Drug as Corrosion Inhibitor for Aluminum in $1 \mathrm{M} \mathrm{HCl}$ Medium: Experimental and Theoretical Studies. IOSR J. Appl. Chem. 11:24-36.

15. Diki NYS, Gbassi GK, Ouedraogo A, Berte M, Trokourey A (2018b). Aluminum corrosion inhibition by cefixime drug: experimental and DFT studies. J. Electrochem. Sci. Eng. 8: 303-320.

16. Durnie W, Marco R.D, Jefferson A, Kinsella B (1999). Development of a Structure-Activity Relationship for Oil Field Corrosion Inhibitors. J. Electrochem. Soc. 146:1751-1756.

17. Ebenso EE, Arslan T, Kandemirli F, Caner IN, Love II (2010). Quantum Chemical Studies of some Rhodamine Azosulpha Drugs as corrosion inhibitors for mild steel in Acidic Medium. Int. J. Quantum Chem. 110:1003-1018.

18. Eddy NO, Stoyanov SR, Ebenso EE (2010). Fluoroquinolones as Corrosion Inhibitors for Mild Steel in Acidic Medium. Int. J. Electrochem. Sci. 5:1127-1150.

19. Ehouman AD, Diomandé GGD, Niamien PM, Sissouma D, Trokourey A (2016). 2-Thiobenzyl-6-methylbenzoxazole as an effective copper corrosion inhibitor in 2M HNO3: experimental and DFT studies. IOSR J. Appl. Chem. 9:17-25.

20. El-Maksoud SAA (2002). Studies on the effect of pyranocoumarin derivatives on the corrosion of iron in $0.5 \mathrm{M} \mathrm{HCl}$. Corros. Sci. 44:803-813.

21. FB, Traisnel M, Lagrenee M (2001). Influence of 2, 5-bis(4dimethylaminophenyl)-1, 3, 4-thiadiazole on corrosion inhibition of mild steel in acidic media. J. Appl. Electrochem. 31:41-48.

22. F., KK (2003). The inhibition of benzimidazole derivatives on corrosion of iron in $1 \mathrm{M} \mathrm{HCl}$ solutions. Electrochim. Acta. 48:2493-2503.

23. Fouda AS, Gouda MM, El-Rahman SIA (2000). Benzaldehyde, 2-hydroxybenzoyl hydrazone derivatives as inhibitors of the corrosion of aluminium in hydrochloric acid. Chem. Pharm. Bull. 48:636-640.

24. Frisch, M.J., Trucks, G.W.,H. B. Schlegel, G.E.S., M. A. Robb, etal., (2009). Revision A.02, Gaussian, Inc., Wallingford CT, 2009.

25. Fuentealba P, Perez P, Contreras R (2000). On the condensed Fukui function. J. Chem. Phys. 113:2544-2551.
26. Gece G (2008). The use of quantum chemical methods in corrosion inhibitor studies. Corros. Sci. 50:2981-2992.

27. Gece G, Bilgic S (2009). Quantum chemical study of some cyclic nitrogen compounds as corrosion inhibitors of steel in $\mathrm{NaCl}$ media. Corros Sci. 51:1876-1878.

28. Hurlen T, Lian H, Odegard OS, Valand TV (1984). Corrosion and passive behaviour of aluminium in weakly acid solution. Electrochim. Acta. 29:579-585.

29. Keles H, Keles M, Dehri I, Serinday O (2008). Adsorption and inhibitive properties of aminobiphenyl and its Schiff base on mild steel corrosion in $0.5 \mathrm{M} \mathrm{HCl}$ medium. Colloids Surf A Physicochem Eng Aspects. 320:138-145.

30. Khaled KF (2008). New Synthesized Guanidine Derivative as a Green Corrosion Inhibitor for Mild Steel in Acidic Solutions. Int. J. Electrochem. Sci. 3:462-475.

31. Khaled KF (2010). Corrosion control of copper in nitric acid solutions using some amino acids-a combined experimental and theoretical study. Corrosi. Sci. 52:3225-3234.

32. Khaled KF, Al-Qahtani MM (2009). The inhibitive effect of some tetrazole derivatives towards Al corrosion in acid solution: Chemical, electrochemical and theoretical studies Mater Chem. Phys. 113:150-158.

33. Khaled KF, Babic-Samardziza K, Hackerman N (2005). Theoretical study of the structural effects of polymethylene amines on corrosion inhibition of iron in acid solutions. Electrochim. Acta. 50:2515-2520.

34. Khaled KF, Hackerman N (2004). Ortho-substituted anilines to inhibit copper corrosion in aerated $0.5 \mathrm{M}$ hydrochloric acid. Electrochim. Acta. 49:485-495.

35. Khalil N (2003). Quantum chemical approach of corrosion inhibition. Electrochim. Acta. 48:2635-2640.

36. Kokalj A, Kovacevic N (2011). On the consistent use of electrophilicity index and HSAB-based electron transfer and its associated change of energy parameters. Chem. Phys. Lett. 507:181-184.

37. Koopmans T (1934). Über die Zuordnung von Wellenfunktionen und Eigenwerten zu den Einzelnen Elektronen Eines Atoms. Physica. 1:104-113.

38. Kouakou V, Niamien PM, Yapo AJ, Trokourey A (2016). Copper Corrosion Inhibition in $1 \mathrm{M}$ Nitric Acid: Adsorption and Inhibitive Action of Theophylline. Chem. Sci. Rev. Lett. 5:131-146.

39. Laarej, K., Bouachrine, M., Radi, S., Kertit, S., Hammouti, B., (2010). EJ. Chem. 7: 419-424.

40. Lebrini M, Lagrenee M, Vezin H, Gengembre L, Bentiss F (2005). Electrochemical and quantum chemical studies of new thiadiazole derivatives adsorption on mild steel in normal hydrochloric acid medium. Corros. Sci. 47:485-505.

41. Lee C, Yang W, Parr RG (1988). Development of the Colle Salvetti correlation-energy formula into a functional of the electron density. Phys. Rev. B. 37:785-789.

42. Lukovits I., Palfi K, Bako I, Kalman E (1997). Corrosion, LKP Model of the Inhibition Mechanism of Thiourea Compounds, Corros. 53:915-919.

43. Luo H, Guan YC, Han KN (1998). Corrosion Inhibition of Mild Steel by Aniline and Alkylamines in Acidic Solutions. Corros. 54:721-731.

44. Martinez-Araya JI (2015). Why is the dual descriptor a more accurate local reactivity descriptor than Fukui functions? J. Math. Chem. 53:451-465. 
45. Michaelson HB (1977). The work function of the elements and its periodicity. J. Appl Phys. 48:4729-4734.

46. Morell C, Grand A, Torro-Labbé A (2005). New Dual Descriptor for Chemical Reactivity. J. Phys. Chem A. 109:205-212.

47. Morell, C., Grand, A., Torro-Labbé, A., (2006). Theoretical support for using the $\Delta \mathrm{f}(\mathrm{r})$ descriptor. Chem. Phys. Lett. 425: 342-346.

48. Musa AY, Kadhum AH, Mohamad AB, Rohoma AB, Mesmari $\mathrm{H}$ (2010). Electrochemical and quantum chemical calculations on 4, 4-dimethyloxazolidine-2-thione as inhibitor for mild steel corrosion in hydrochloric acid. J. Mol. Struct. 969:233-237.

49. Obot IB, Obi-Egbedi NO, Umoren SA (2009). Adsorption Characteristics and Corrosion Inhibitive Properties of Clotrimazole for Aluminium Corrosion in Hydrochloric Acid. Int. J. Electrochem. Sci. 4: 863 - 877.

50. Obot IB, Umoren SA, Obi-Egbedi NO (2011). Corrosion inhibition and adsorption behaviour for aluminuim by extract of Aningeria robusta in $\mathrm{HCl}$ solution: Synergistic effect of iodide ions. J. Mater. Environ. Sci. 2:60-71.

51. Oguzie EE, Akalezi CO, Enenebeaku CK, Aneke JN (2011a). Corrosion inhibition and adsorption behaviour of malachite green dye on aluminium corrosion. Chem. Eng. Commun. 198:46-60.

52. Oguzie EE, Li Y, Wang SG (2011b). Understanding corrosion inhibition mechanisms-experimental and theoretical approach. RSC Adv. 1:866-873.

53. Ouédraogo A, Akpa SJ, Diki NYS, Diomandé GGD, Coulibaly NH, Trokourey A (2018). 7-(2-Ethyltiophenyl) Theophylline as Copper Corrosion Inhibitor in $1 \mathrm{M}$ HNO3. J. Mater. Sci. Chem. Eng. 6:31-49.

54. Özcan M, Dehri I (2004). Electrochemical and quantum chemical studies of some sulphur-containing organic compounds as inhibitors for the acid corrosion of mild steel. Prog. Org. Coat. 51:181-187.

55. Parr RG, Donnelly RA, Levy M, Palke WE (1978). Electronegativity: The density functional viewpoint. J. Chem. Phys. 68:3801-3807.

56. Parr, R.G., Pearson, R.G., (1983). Absolute hardness: Companion parameter to absolute electronegativity. J. Am. Chem. Soc. 105: 7512-7516.

57. Parr, R.G., Szentpaly, L.V., Liu, S., (1999). Electrophilicity index. J. Am. Chem. Soc. 121: 1922-1924.

58. Patel NS, Beranek P, Nebyla M, Pribyl M, Snita D (2014). Inhibitive Effects by Some Benzothiazole Derivatives on Mild Steel Corrosion in $1 \mathrm{~N} \mathrm{HCl}$. Int. J. Electrochem. Sci. 9:3951-3960.

59. Popova A, Christov M, Deligeorgiev T (2003). Influence of the Molecular Structure on the Inhibitor Properties of Benzimidazole Derivatives on Mild Steel Corrosion in $1 \mathrm{M}$ Hydrochloric Acid. Corros. 59:756-764.

60. Quraishi MA, Singh A, Singh VK, Yadav DK, Singh AK, (2010). Green approach to corrosion inhibition of mild steel in hydrochloric acid and sulphuric acid solutions by the extract of Murraya koenigii leaves. Mat. Chem. phys. 22:114-122.
61. Rodriguez-Valdez LM, Martinez-Villafane A, GlossmanMitnik D (2005). CHIH-DFT theoretical study of isomeric thiatriazoles and their potential activity as corrosion inhibitors. J. Mol. Struct. : (Theochem). 716:61-65.

62. Santhini N, Jeyaraj T (2012). The inhibition effect of [3-(4-hydroxy-3-methoxy-phenyl)-1- phenyl-propenone] on the corrosion of the aluminium in alkaline medium. J. Chem. Pharm. Res. 4:3550-3556.

63. Saratha R, Meenakshi R (2010). Corrosion inhibitor: A plant extract. Pharma Chem. 2:287-294.

64. Sastri VS, Perumareddi JR (1997). Molecular Orbital Theoretical Studies of Some Organic Corrosion Inhibitors. Corros. 53:617-622.

65. Satapathy AK, Gunasekaran G, Sahoo SC, Amit K, Rodrigues PV (2009). Corrosion inhibition by Justicia gendarussa plant extract in hydrochloric acid solution. Corros. Sci. 51:2848-2856.

66. Scendo M, Hepel M (2008). Inhibiting properties of benzimidazole films for $\mathrm{Cu}(\mathrm{II}) / \mathrm{Cu}(\mathrm{I})$ reduction in chloride media studied by RDE and EQCN techniques. J. Electroanal.Chem. 613:35-50

67. Singh A, Ahamad I, Quraishi MA (2016). Piper longum extract as green corrosion inhibitor for aluminium in $\mathrm{NaOH}$ solution. Arabian J. Chem. 9:S1584-S1589.

68. Udhayakala P, Rajendiran TV (2015). A theoretical evaluation on benzothiazole derivatives as corrosion inhibitors on mild Steel. Pharma Chem. 7:92-99.

69. Udhayakala P, Rajendiran TV, Gunasekaran S (2012). Theoretical approach to the corrosion inhibition efficiency of some pyrimidine derivatives using DFT method. J. Comput. Methods Mol. Des. 2:1-15.

70. Umoren SA (2008). Inhibition of aluminium and mild steel corrosion in acidic medium using gum Arabic. Cellulose. 15:751-761.

71. Umoren SA, Obot IB, Obi-Egbedi NO (2009). Raphia hookeri gum as a potential eco-friendly inhibitor for mild steel in sulfuric acid. J. Mater. Sci. 44:274-279.

72. Villamil RFV, Corio P, Rubin JC, Agostinho SML (1999). Effect of sodium dodecylsulfate on copper corrosion in sulfuric acid media in the absence and presence of benzotriazole. J. Electroanal. Chem. 472:112-116.

73. Yeo M, Niamien PM, Bilé EBA, Trokourey A (2017). Thiamine Hydrochloride as a Potential Inhibitor for Aluminium Corrosion in 1.0M HCl: Mass Loss and DFT Studies. J. Comput. Methods Mol. Des. 7:13-25.

How to cite article: (2018). N. Y. S. Diki , Gbe Gondo Didier Diomandé, Sagne Jacques Akpa, Augustin Ouédraogo, et al., (2018). Aluminium Corrosion Inhibition by 7-(Ethylthiobenzimidazolyl) Theophylline in $1 \mathrm{M}$ Hydrochloric Acid : Experimental and DFT Studies. Int. J. Appl. Pharm. Sci. Res. 3(4):41-53. doi: https://doi.org/10.21477/ijapsr.3.4.1

\section{Source of Support; Nil}

Conflict of Interest: None 